\title{
Anecdotes of lithogenesis and atherogenesis conversely liable for cardiac dysfunction and kidney stone formation
}

\author{
Asokan Devarajan
}

Received: 18 December 2014/ Accepted: 27 January 2015/Published online: 8 February 2015

(C) Springer-Verlag Berlin Heidelberg 2015

\section{Dear Editor,}

Recently, Masterson et al. [1] reported that dyslipidemia increases the risk of kidney stone formation. Interestingly, in 2002 Khan et al. reported [2] that urolithic patients excrete more lipids such as total cholesterol, triglycerides, and some phospholipids [2]. The same study further suggested that lipids modulate the calcium oxalate crystal nucleation, aggregation and retention processes, which are the important steps for kidney stone development [2].

On the other hand, the association of kidney stones and subclinical carotid atherosclerosis has been observed in young adults implying that renal lithogenesis and atherogenesis share common pathophysiology both systemically and on a cellular level [3], where oxalate would act as a pro-atherogenic factor to increase the oxidative stress [2] as seen in atherosclerosis patients [3]. Injury and retention model explains the lithogenesis and atherogenesis [2, 3]. In kidney stones, retention of calcium oxalate crystals occurs in the injured urothelium, inducing accumulation of lipids, proteinaceous materials, such as calcium-binding protein and glycoproteins, which interact with crystals and modulate the nucleation and aggregation process leading to stone formation [1]. Vascular cell injury and retention of lipids in the arterial wall cause the atherosclerosis [3]. In humans, all these processes take place over a long period of time $[2,3]$ during which several metabolic changes occur finally ending in lithogenesis and switching on atherogenesis, or vice versa.

\footnotetext{
A. Devarajan $(\bowtie)$

Departments of Obstetrics and Gynecology, David Geffen School of Medicine, University of California, 650 Charles E. Young drive South, CHS B8-127, Los Angeles, CA 90095, USA e-mail: adevarajan@mednet.ucla.edu
}

Thus, even though patients have either kidney stone alone or cardiovascular disease alone, it would be ideal for physicians to screen for risk factors of both diseases periodically. Metabolic changes can also be altered due to transient food consumption. For example, it is well accepted that oxalate and purine rich foods can lead to hyperoxaluria and hyperuricosuria, respectively, while oil rich food increases plasma cholesterol and triglycerides levels. Hence, to avoid a false diagnosis, it would be better to measure within different time frames. Further, identification of drugs inhibiting both atherogenesis and lithogenesis would enable the medical community to ease the pain of patients and be economically conservative.

\section{Sincerely \\ Asokan Devarajan}

Acknowledgments I thank Dr. Maria Johnson and Maria Yesayan for editing the manuscript.

Conflict of interest There are no conflicts of interest.

\section{References}

1. Masterson JH, Woo JR, Chang DC, Chi T, L'Esperance JO, Stoller ML, Sur RL (2014) Dyslipidemia is associated with an increased risk of nephrolithiasis Urolithiasis [Epub ahead of print]

2. Khan SR, Glenton PA, Backov R, Talham DR (2002) Presence of lipids in urine, crystals and stones: implications for the formation of kidney stones. Kidney Int 62:2062-2072

3. Reiner AP, Kahn A, Eisner BH, Pletcher MJ, Sadetsky N, Williams OD, Polak JF, Jacobs DR Jr, Stoller ML (2011) Kidney stones and subclinical atherosclerosis in young adults: the CARDIA study. J Urol 185:920-925 